TAIWANESE JOURNAL OF MATHEMATICS

Vol. 15, No. 2, pp. 787-818, April 2011

This paper is available online at http://www.tjm.nsysu.edu.tw/

\title{
NONLINEAR OPERATORS OF MONOTONE TYPE AND CONVERGENCE THEOREMS WITH EQUILIBRIUM PROBLEMS IN BANACH SPACES
}

\author{
Wataru Takahashi and Jen-Chih Yao*
}

\begin{abstract}
Our purpose in this paper is first to discuss nonlinear operators and nonlinear projections in Banach spaces which are related to the resolvents of $m$-accretive operators and maximal monotone operators. Some of these operators in Banach spaces are new. Next, we discuss some properties for such nonlinear operators and nonlinear projections in Banach spaces. Further, using these properties, we prove strong convergence theorems by hybrid methods for nonlinear operators with equilibrium problems in Banach spaces.
\end{abstract}

\section{INTRODUCTION}

Let $E$ be a Banach space and let $E^{*}$ be the dual space of $E$. Let $A: E \rightarrow 2^{E}$ be a multi-valued operator with domain $D(A)=\{z \in E: A z \neq \emptyset\}$ and range $R(A)=\cup\{A z: z \in D(A)\}$. Then, $A$ is called accretive if for each $x_{i} \in D(A)$ and $y_{i} \in A x_{i}, i=1,2$, there exists $j \in J\left(x_{1}-x_{2}\right)$ such that $\left\langle y_{1}-y_{2}, j\right\rangle \geq 0$, where $J$ is the duality mapping from $E$ into $2^{E^{*}}$ An accretive operator $A$ is $m$ accretive if and only if $R(I+r A)=E$ for all $r>0$. If $A$ is $m$-accretive, then for each $r>0$ and $x \in E$, we can define the resolvent $J_{r}: R(I+r A) \rightarrow D(A)$ by $J_{r} x=\{z \in E: x \in z+r A z\}$. A multi-valued operator $A: E \rightarrow 2^{E^{*}}$ with domain $D(A)=\{z \in E: A z \neq \emptyset\}$ and range $R(A)=\cup\{A z: z \in D(A)\}$ is said to be monotone if $\left\langle x_{1}-x_{2}, y_{1}-y_{2}\right\rangle \geq 0$ for each $x_{i} \in D(A)$ and $y_{i} \in A x_{i}, i=1,2$. The monotone operator $A$ is said to be maximal if its graph $G(A)=\{(x, y): y \in A x\}$ is not properly contained in the graph of any other monotone operator. Let $E$ be a reflexive, strictly convex and smooth Banach space and let $A: E \rightarrow 2^{E^{*}}$ be a monotone operator. Then, $A$ is maximal if and only if $R(J+r A)=E^{*}$ for all

Received October 21, 2009.

2000 Mathematics Subject Classification: 47H05, 47H09, 47H20.

Key words and phrases: Nonlinear mapping, Fixed point, Maximal monotone operator, Accretive operator, Resolvent, Duality theorem.

*Corresponding author. 
$r>0$; see [47]. If $A: E \rightarrow 2^{E^{*}}$ is a maximal monotone operator, then for $\lambda>0$ and $x \in E$, we can consider the following resolvents:

$$
J_{\lambda} x=\{z \in E: 0 \in J(z-x)+\lambda A(z)\}
$$

and

$$
Q_{\lambda} x=\{z \in E: J x \in J z+\lambda A(z)\} .
$$

Further, if $B: E^{*} \rightarrow 2^{E}$ be a maximal monotone operator, then for $\lambda>0$ and $x \in E$, we can consider the resolvent

$$
R_{\lambda} x=\{z \in E: x \in z+\lambda B J(z)\} .
$$

These four resolvents are important and have interesting properties.

Let $C$ be a nonempty closed convex subset of a Banach space $E$ and let $f$ : $C \times C \rightarrow \mathbb{R}$ be a bifunction. We consider the following equilibrium problem:

$$
\text { Find } z \in C \text { such that } f(z, y) \geq 0, \quad \forall y \in C \text {. }
$$

The set of such $z \in C$ is denoted by $E P(f)$, i.e.,

$$
E P(f)=\{z \in C: f(z, y) \geq 0, \quad \forall y \in C\} .
$$

Problem (1.1) is also important in the sense that it includes, as special cases, optimization problems, variational inequalities, minimax problems, Nash equilibrium problem in noncooperative games and others; see, for instance, [32] and [6].

Our purpose in this paper is first to discuss nonlinear operators and nonlinear projections in Banach spaces which are related to the resolvents of $m$-accretive operators and maximal monotone operators. Some of these operators are new. Next, we discuss fixed point theorems and duality theorems for such nonlinear operators and nonlinear projections in Banach spaces. Further, using these properties, we prove strong convergence theorems by hybrid methods for nonlinear operators with equilibrium problems in Banach spaces.

\section{PReLIMINARIES}

Let $E$ be a real Banach space with norm $\|\cdot\|$ and let $E^{*}$ be the dual of $E$. We denote the value of $y^{*} \in E^{*}$ at $x \in E$ by $\left\langle x, y^{*}\right\rangle$. When $\left\{x_{n}\right\}$ is a sequence in $E$, we denote the strong convergence of $\left\{x_{n}\right\}$ to $x \in E$ by $x_{n} \rightarrow x$ and the weak convergence by $x_{n} \rightarrow x$. The modulus $\delta$ of convexity of $E$ is defined by

$$
\delta(\epsilon)=\inf \left\{1-\frac{\|x+y\|}{2}:\|x\| \leq 1,\|y\| \leq 1,\|x-y\| \geq \epsilon\right\}
$$


for every $\epsilon$ with $0 \leq \epsilon \leq 2$. A Banach space $E$ is said to be uniformly convex if $\delta(\epsilon)>0$ for every $\epsilon>0$. A uniformly convex Banach space is strictly convex and reflexive. Let $C$ be a nonempty closed convex subset of a strictly convex and reflexive Banach space $E$. Then we know that for any $x \in E$, there exists a unique element $z \in C$ such that $\|x-z\| \leq\|x-y\|$ for all $y \in C$. Putting $z=P_{C}(x)$, we call $P_{C}$ the metric projection of $E$ onto $C$. The duality mapping $J$ from $E$ into $2^{E^{*}}$ is defined by

$$
J x=\left\{x^{*} \in E^{*}:\left\langle x, x^{*}\right\rangle=\|x\|^{2}=\left\|x^{*}\right\|^{2}\right\}
$$

for every $x \in E$. Let $U=\{x \in E:\|x\|=1\}$. The norm of $E$ is said to be Gateaux differentiable if for each $x, y \in U$, the limit

$$
\lim _{t \rightarrow 0} \frac{\|x+t y\|-\|x\|}{t}
$$

exists. In the case, $E$ is called smooth. We know that $E$ is smooth if and only if $J$ ia a single valued mapping of $E$ into $E^{*}$. We also know that $E$ is reflexive if and only if $J$ is surjective, and $E$ is strictly convex if and only if $J$ is one-to-one. Therefore, if $E$ is a smooth, strictly convex and reflexive Banach space, then $J$ is a single-valued bijection and in this case, the inverse mapping $J^{-1}$ coincides with the duality mapping $J_{*}$ on $E^{*}$. The norm of $E$ is said to be uniformly Gateaux differentiable if for each $y \in U$, the limit (2.1) is attained uniformly for $x \in U$. It is also said to be Fréchet differentiable if for each $x \in U$, the limit (2.1) is attained uniformly for $y \in U$. A Banach space $E$ is called uniformly smooth if the limit (2.1) is attained uniformly for $x, y \in U$. It is known that if the norm of $E$ is uniformly Gateaux differentiable, then $J$ is uniformly norm to weak ${ }^{*}$ continuous on each bounded subset of $E$, and if the norm of $E$ is Frechet differentiable, then $J$ is norm to norm continuous. If $E$ is uniformly smooth, $J$ is uniformly norm to norm continuous on each bounded subset of $E$. For more details, see [46].

We know the following result: Let $E$ be a smooth, strictly convex and reflexive Banach space. Let $C$ be a nonempty closed convex subset of $E$ and let $P_{C}$ be the metric projection of $E$ onto $C$. Let $x_{0} \in C$ and $x_{1} \in E$. Then, $x_{0}=P_{C}\left(x_{1}\right)$ if and only if

$$
\left\langle x_{0}-y, J\left(x_{1}-x_{0}\right)\right\rangle \geq 0
$$

for all $y \in C$, where $J$ is the duality mapping of $E$.

Let $C$ be a nonempty subset of $E$ and let $T$ be a mapping of $C$ into $E$. We denote the set of all fixed points of $T$ by $F(T)$. A mapping $T: C \rightarrow E$ is said to be nonexpansive if $\|T x-T y\| \leq\|x-y\|$ for all $x, y \in C$. Let $D$ be a subset of $C$ and let $P$ be a mapping of $C$ into $D$. Then $P$ is said to be sunny if

$$
P(P x+t(x-P x))=P x
$$


whenever $P x+t(x-P x) \in C$ for $x \in C$ and $t \geq 0$. $A$ mapping $P$ of $C$ into $C$ is said to be a retraction if $P^{2}=P$. We denote the closure of the convex hull of $D$ by $\overline{\mathrm{co}} D$.

Let $E$ be a Banach space and let $A: E \rightarrow 2^{E}$ be a multi-valued operator. Then, $A$ is called accretive if for each $x_{i} \in D(A)$ and $y_{i} \in A x_{i}, i=1,2$, there exists $j \in J\left(x_{1}-x_{2}\right)$ such that $\left\langle y_{1}-y_{2}, j\right\rangle \geq 0$. An accretive operator $A$ is $m$-accretive if and only if $R(I+r A)=E$ for all $r>0$. If $A$ is $m$-accretive, then for each $r>0$ and $x \in E$, we can define $J_{r}: R(I+r A) \rightarrow D(A)$ by $J_{r} x=\{z \in E: x \in z+r A z\}$. We call such $J_{r}=(I+r A)^{-1}$ the accretive resolvent of $A$ for $r>0$.

A multi-valued operator $A: E \rightarrow 2^{E^{*}}$ with domain $D(A)=\{z \in E: A z \neq \emptyset\}$ and range $R(A)=\bigcup\{A z: z \in D(A)\}$ is said to be monotone if $\left\langle x_{1}-x_{2}, y_{1}-y_{2}\right\rangle \geq$ 0 for each $x_{i} \in D(A)$ and $y_{i} \in A x_{i}, i=1,2$. A monotone operator $A$ is said to be maximal if its graph $G(A)=\{(x, y): y \in A x\}$ is not properly contained in the graph of any other monotone operator. The following theorems are well known; see, for instance, [47].

Theorem 2.1. Let $E$ be a reflexive, strictly convex and smooth Banach space and let $A: E \rightarrow 2^{E^{*}}$ be a monotone operator. Then $A$ is maximal if and only if $R(J+r A)=E^{*}$ for all $r>0$.

Theorem 2.2. Let $E$ be a smooth Banach space and let $J$ be the duality mapping on $E$. Then, $\langle x-y, J x-J y\rangle \geq 0$ for all $x, y \in E$. Further, if $E$ is strictly convex and $\langle x-y, J x-J y\rangle=0$, then $x=y$.

Let $E$ be a reflexive, strictly convex and smooth Banach space and let $A: E \rightarrow$ $2^{E^{*}}$ be a maximal monotone operator. Then, for $\lambda>0$ and $x \in E$, consider

$$
J_{\lambda} x=\{z \in E: 0 \in J(z-x)+\lambda A(z)\}
$$

and

$$
Q_{\lambda} x=\{z \in E: J x \in J z+\lambda A(z)\} .
$$

We denote $J_{\lambda}$ and $Q_{\lambda}$ by $J_{\lambda}=\left(I+\lambda J^{-1} A\right)^{-1}$ and $Q_{\lambda}=(J+\lambda A)^{-1} J$, respectively. We call such $J_{\lambda}$ and $Q_{\lambda}$ the metric resolvent and the relative resolvent of $A$ for $\lambda>0$, respectively. We also consider another resolvent of a maximal monotone operator. Let $B: E^{*} \rightarrow 2^{E}$ be a maximal monotone operator. Then, for $\lambda>0$ and $x \in E$, consider

$$
R_{\lambda} x=\{z \in E: x \in z+\lambda B J(z)\} .
$$

We denote $R_{\lambda}$ by $R_{\lambda}=(I+\lambda B J)^{-1}$. We call such $R_{\lambda}$ the generalized resolvent of $B$ for $\lambda>0$. 
Let $E$ be a reflexive, strictly convex and smooth Banach space. The function $\phi: E \times E \rightarrow(-\infty, \infty)$ is defined by

$$
\phi(x, y)=\|x\|^{2}-2\langle x, J y\rangle+\|y\|^{2}
$$

for $x, y \in E$, where $J$ is the duality mapping of $E$; see [1] and [20]. We have from the definition of $\phi$ that

$$
\phi(x, y)=\phi(x, z)+\phi(z, y)+2\langle x-z, J z-J y\rangle
$$

for all $x, y, z \in E$. From $\left(\|x\|^{2}-\|y\|^{2}\right) \leq \phi(x, y)$ for all $x, y \in E$, we can see that $\phi(x, y) \geq 0$. Let $\phi_{*}: E^{*} \times E^{*} \rightarrow(-\infty, \infty)$ be the function defined by

$$
\phi_{*}\left(x^{*}, y^{*}\right)=\left\|x^{*}\right\|^{2}-2\left\langle J^{-1} y^{*}, x^{*}\right\rangle+\left\|y^{*}\right\|^{2}
$$

for $x^{*}, y^{*} \in E^{*}$, where $J$ is the duality mapping of $E$. It is easy to see that

$$
\phi(x, y)=\phi_{*}(J y, J x)
$$

for $x, y \in E$. If $E$ is additionally assumed to be strictly convex, then

$$
\phi(x, y)=0 \Longleftrightarrow x=y .
$$

If $C$ is a nonempty closed convex subset of a smooth, strictly and reflexive Banach space $E$, then for all $x \in E$ there exists a unique $z \in C$ (denoted by $\left.\Pi_{C} x\right)$ such that

$$
\phi(z, x)=\min _{y \in C} \phi(y, x) .
$$

The mapping $\Pi_{C}$ is called the generalized projection from $E$ onto $C$; see Alber [1], Alber and Reich [2], and Kamimura and Takahashi [20].

The following theorem is well known; see, for instance, [20].

Theorem 2.3. Let $E$ be a reflexive, strictly convex and smooth Banach space and let $\left\{x_{n}\right\}$ and $\left\{y_{n}\right\}$ be sequences in $E$ such that $\left\{x_{n}\right\}$ or $\left\{y_{n}\right\}$ is bounded. If $\lim _{n \rightarrow \infty} \phi\left(x_{n}, y_{n}\right)=0$, then $\lim _{n \rightarrow \infty}\left\|x_{n}-y_{n}\right\|=0$.

For a sequence $\left\{C_{n}\right\}$ of nonempty closed convex subsets of a reflexive Banach space $E$, define s-Li $C_{n}$ and w-Ls $C_{n}$ as follows: $x \in \mathrm{s}-\mathrm{Li}_{n} C_{n}$ if and only if there exists $\left\{x_{n}\right\} \subset E$ such that $\left\{x_{n}\right\}$ converges strongly to $x$ and that $x_{n} \in C_{n}$ for all $n \in \mathbb{N}$. Similarly, $y \in \mathrm{w}-\operatorname{Ls}_{n} C_{n}$ if and only if there exist a subsequence $\left\{C_{n_{i}}\right\}$ of $\left\{C_{n}\right\}$ and a sequence $\left\{y_{i}\right\} \subset E$ such that $\left\{y_{i}\right\}$ converges weakly to $y$ and that $y_{i} \in C_{n_{i}}$ for all $i \in \mathbb{N}$. If $C_{0}$ satisfies that

$$
C_{0}=\mathrm{s}-\mathrm{Li}_{n} C_{n}=\mathrm{w}-\mathrm{Ls}_{n} C_{n},
$$


it is said that $\left\{C_{n}\right\}$ converges to $C_{0}$ in the sense of Mosco [30] and we write $C_{0}=\mathrm{M}-\lim _{n \rightarrow \infty} C_{n}$. It is easy to show that if $\left\{C_{n}\right\}$ is nonincreasing with respect to inclusion, then $\left\{C_{n}\right\}$ converges to $\cap_{n=1}^{\infty} C_{n}$ in the sense of Mosco. For more details, see [30]. We know the following theorem [11].

Theorem 2.4. Let $E$ be a smooth Banach space and let $E^{*}$ have a Frechet differentiable norm. Let $\left\{C_{n}\right\}$ be a sequence of nonempty closed convex subsets of $E$. If $C_{0}=M-\lim _{n \rightarrow \infty} C_{n}$ exists and nonempty, then for each $x \in E, \Pi_{C_{n}} x$ converges strongly to $\Pi_{C_{0}}$ x, where $\Pi_{C_{n}}$ and $\Pi_{C_{0}}$ are the generalized projections of $E$ onto $C_{n}$ and $C_{0}$, respectively.

Let $C$ be a nonempty closed subset of a smooth, strictly convex and reflexive Banach space $E$ such that $J C$ is closed and convex. For solving the equilibrium problem, let us assume that a bifunction $f: J C \times J C \rightarrow \mathbb{R}$ satisfies the following conditons:

(A1) $f\left(x^{*}, x^{*}\right)=0, \quad \forall x^{*} \in J C$;

(A2) $f$ is monotone, i.e., $f\left(x^{*}, y^{*}\right)+f\left(y^{*}, x^{*}\right) \leq 0, \quad \forall x^{*}, y^{*} \in J C$;

(A3) $\lim _{t \downarrow 0} f\left(t z^{*}+(1-t) x^{*}, y^{*}\right) \leq f\left(x^{*}, y^{*}\right), \quad \forall x^{*}, y^{*}, z^{*} \in J C$;

(A4) for each $x^{*} \in J C, y^{*} \mapsto f\left(x^{*}, y^{*}\right)$ is convex and lower semicontinuous.

The following result is in Takahashi and Zembayashi [57]; see also Blum and Oettli [6] and Aoyama, Kimura and Takahashi [3].

Lemma 2.5. Let $C$ be a nonempty closed subset of a smooth, strictly convex and reflexive Banach space $E$ such that $J C$ is closed and convex, let $f$ be a bifunction from $J C \times J C$ into $\mathbb{R}$ satisfying (A1), (A2), (A3) and (A4). Then, for any $r>0$ and $x \in E$, there exists a unique $z \in C$ such that

$$
f(J z, J y)+\frac{1}{r}\langle J y-J z, z-x\rangle \geq 0, \quad \forall y \in C .
$$

Further, define $T_{r} x=\left\{z \in C: f(J z, J y)+\frac{1}{r}\langle J y-J z, z-x\rangle \geq 0, \forall y \in C\right\}$ for all $r>0$ and $x \in H$. Then the following hold:

(1) $T_{r}$ is single-valued;

(2) $T_{r}$ is firmly generalized nonexpansive, i.e.,

$$
\left\langle T_{r} x-T_{r} y, J T_{r} x-J T_{r} y\right\rangle \leq\left\langle x-y, J T_{r} x-J T_{r} y\right\rangle, \quad \forall x, y \in E ;
$$

(3) $F\left(T_{r}\right)=E P(f)$

(4) JEP $(f)$ is closed and convex;

(5) $\phi\left(x, T_{r} x\right)+\phi\left(T_{r} x, q\right) \leq \phi(x, q), \quad \forall x \in E, q \in F\left(T_{r}\right)$. 


\section{Four Nonlinear Retractions}

In this section, we first define nonlinear operators which are deduced from $m$ accretive operators and maximal monotone operators in a Banach space. If $A: E \rightarrow$ $2^{E}$ is $m$-accretive, then for each $\lambda>0$ and $x \in E$, we can define the accretive resolvent $J_{\lambda}: E \rightarrow D(A)$ by $J_{\lambda} x=\{z \in E: x \in z+\lambda A z\}$. Then, we know that $J_{\lambda} x$ is always nonempty and a singleton. We denote such $J_{\lambda}$ by $J_{\lambda}=(I+\lambda A)^{-1}$. If $J_{\lambda}$ is the accretive resolvent, then we can show that

$$
0 \leq\left\langle x-J_{\lambda} x-\left(y-J_{\lambda} y\right), J\left(J_{\lambda} x-J_{\lambda} y\right)\right\rangle
$$

for all $x, y \in E$. Let $C$ be a subset of $E$. Then, a nonlinear operator $T: C \rightarrow E$ is called firmly nonexpansive if

$$
0 \leq\langle x-T x-(y-T y), J(T x-T y)\rangle
$$

for all $x, y \in C$. If $A: E \rightarrow 2^{E^{*}}$ is a maximal monotone operator, then for $\lambda>0$ and $x \in E$, we define the metric resolvent $J_{\lambda}: E \rightarrow D(A)$ by $J_{\lambda} x=\{z \in E: 0 \in$ $J(z-x)+\lambda A(z)\}$. Then, we know that $J_{\lambda} x$ is always nonempty and a singleton. We denote such $J_{\lambda}$ by $J_{\lambda}=\left(I+\lambda J^{-1} A\right)^{-1}$. If $J_{\lambda}$ is the metric resolvent, then we have

$$
0 \leq\left\langle J_{\lambda} x-J_{\lambda} y, J\left(x-J_{\lambda} x\right)-J\left(y-J_{\lambda} y\right)\right\rangle
$$

for all $x, y \in E$; see, for instance, [4]. In general, a nonlinear operator $T: C \rightarrow E$ is called firmly metric if

$$
0 \leq\langle T x-T y, J(x-T x)-J(y-T y)\rangle
$$

for all $x, y \in C$. If $A: E \rightarrow 2^{E^{*}}$ is a maximal monotone operator, then for $\lambda>0$ and $x \in E$, we can consider the relative resolvent $Q_{\lambda}: E \rightarrow D(A)$ by $Q_{\lambda} x=\{z \in E: J x \in J z+\lambda A(z)\}$. Then, we know that $Q_{\lambda} x$ is always nonempty and a singleton. We denote such $Q_{\lambda}$ by $Q_{\lambda}=(J+\lambda A)^{-1} J$. If $Q_{\lambda}$ is the relative resolvent, then we have

$$
0 \leq\left\langle J_{\lambda} x-J_{\lambda} y, J x-J J_{\lambda} x-\left(J y-J J_{\lambda} y\right)\right\rangle
$$

for all $x, y \in E$. In general, a nonlinear operator $T: C \rightarrow E$ is firmly relative nonexpansive if

$$
0 \leq\langle T x-T y, J x-J T x-(J y-J T y)\rangle
$$

for all $x, y \in C$. We can define another nonlinear operator. If $B: E^{*} \rightarrow 2^{E}$ is a maximal monotone operator, then for $\lambda>0$ and $x \in E$, we can consider the generalized resolvent $R_{\lambda}: E \rightarrow D(A)$ by $R_{\lambda} x=\{z \in E: x \in z+\lambda B J(z)\}$. 
Then, we know that $R_{\lambda} x$ is always nonempty and a singleton. We denote such $R_{\lambda}$ by $R_{\lambda}=(I+\lambda B J)^{-1}$. If $R_{\lambda}$ is the generalized resolvent, then we know that

$$
0 \leq\left\langle x-J_{\lambda} x-\left(y-J_{\lambda} y\right), J J_{\lambda} x-J J_{\lambda} y\right\rangle
$$

for all $x, y \in E$. In general, a nonlinear operator $T: C \rightarrow E$ is firmly generalized nonexpansive if

$$
0 \leq\langle x-T x-(y-T y), J T x-J T y\rangle
$$

for all $x, y \in C$.

Next, we define four projections in a Banach space. Let $E$ be a reflexive, smooth and strictly convex Banach space. We know that $T: C \rightarrow E$ is firmly nonexpansive if

$$
0 \leq\langle x-T x-(y-T y), J(T x-T y)\rangle
$$

for all $x, y \in C$. If $F(T)$ is nonempty, then we have that

$$
0 \leq\langle x-T x, J(T x-y)\rangle
$$

for all $x \in C$ and $y \in F(T)$. Let $P$ be a retraction of $E$ onto $C$, i.e., $P^{2}=P$ and $P(E)=C$. Then a retraction $P$ is called sunny nonexpansive if

$$
0 \leq\langle x-P x, J(P x-y)\rangle
$$

for all $x \in E$ and $y \in C$. We know that $T: C \rightarrow E$ is a firmly metric operator if

$$
0 \leq\langle T x-T y, J(x-T x)-J(y-T y)\rangle
$$

for all $x, y \in C$. If $F(T)$ is nonempty, then we have that

$$
0 \leq\langle T x-y, J(x-T x)\rangle
$$

for all $x \in C$ and $y \in F(T)$. A retraction $P$ of $E$ onto $C$ is called metric if

$$
0 \leq\langle P x-y, J(x-P x)\rangle
$$

for all $x \in E$ and $y \in C$. If $T: C \rightarrow E$ is firmly relative nonexpansive, then we have

$$
0 \leq\langle T x-T y, J x-J T x-(J y-J T y)\rangle
$$

for all $x, y \in C$. If $F(T)$ is nonempty, then we have that

$$
0 \leq\langle T x-y, J x-J T x\rangle
$$


for all $x \in C$ and $y \in F(T)$. A retraction $\Pi_{C}$ of $E$ onto $C$ is called generalized if

$$
0 \leq\left\langle\Pi_{C} x-y, J x-J \Pi_{C} x\right\rangle
$$

for all $x \in E$ and $y \in C$. Such a retraction is also the generalized projection; see $[1,2]$. If $T: C \rightarrow E$ is firmly generalized nonexpansive, we have

$$
0 \leq\langle x-T x-(y-T y), J T x-J T y\rangle
$$

for all $x, y \in C$. If $F(T)$ is nonempty, then we have

$$
0 \leq\langle x-T x, J T x-J y\rangle
$$

for all $x \in C$ and $y \in F(T)$. A retraction $R$ of $E$ onto $C$ is called sunny generalized nonexpansive if

$$
0 \leq\langle x-R x, J R x-J y\rangle
$$

for all $x \in E$ and $y \in C$; see also [12].

Kohsaka and Takahashi [24] proved the following theorems.

Theorem 3.1. (Kohsaka and Takahashi [24]). Let E be a smooth, strictly convex and reflexive Banach space and let $C_{*}$ be a nonempty closed convex subset of $E^{*}$. Suppose that $\Pi_{C_{*}}$ is the generalized projection of $E^{*}$ onto $C_{*}$. Then, $R$ defined by $R=J^{-1} \Pi_{C_{*}} J$ is a sunny generalized nonexpansive retraction of $E$ onto $J^{-1} C_{*}$.

Theorem 3.2. (Kohsaka and Takahashi [24]). Let E be a smooth, strictly convex and reflexive Banach space and let $D$ be a nonempty subset of $E$. Then, the following conditions are equivalent

(1) $D$ is a sunny generalized nonexpansive retract of $E$;

(2) $D$ is a generalized nonexpansive retract of $E$;

(3) JD is closed and convex.

In this case, $D$ is closed.

Theorem 3.3. (Kohsaka and Takahashi [24]). Let E be a smooth, strictly convex and reflexive Banach space and let $D$ be a nonempty closed subset of $E$. Suppose that there exists a sunny generalized nonexpansive retraction $R$ of $E$ onto $D$ and let $(x, z) \in E \times C$. Then, the following conditions are equivalent

(1) $z=R x$;

(2) $\phi(x, z)=\min _{y \in D} \phi(x, y)$.

Ibaraki and Takahashi [12] also proved the following theorems. 
Theorem 3.4. (Ibaraki and Takahashi [12]). Let $E$ be a smooth, strictly convex and reflexive Banach space and let $D$ be a nonempty closed subset of E. Then, a sunny generalized nonexpansive retraction of $E$ onto $D$ is uniquely determined.

Theorem 3.5. (Ibaraki and Takahashi [12]). Let $E$ be a smooth, strictly convex and reflexive Banach space and let $D$ be a nonempty closed subset of E. Suppose that there exists a sunny generalized nonexpansive retraction $R$ of $E$ onto $D$ and let $(x, z) \in E \times C$. Then, the following hold:

(1) $z=R x$ if and only if $\langle x-z, J y-J z\rangle \leq 0, \forall y \in D$;

(2) $\phi(R x, z)+\phi(x, R x) \leq \phi(x, z)$.

\section{Four NONLinear Operators}

Let $E$ be a reflexive, smooth and strictly convex Banach space. Let $C$ be a nonempty subset of $E$. If $T: C \rightarrow E$ is a firmly nonexpansive mapping, then we have that for any $x, y \in C$,

$$
\begin{aligned}
0 & \leq\langle x-T x-(y-T y), J(T x-T y)\rangle \\
& \Longleftrightarrow\|T x-T y\|^{2} \leq\langle x-y, J(T x-T y)\rangle \\
& \Longleftrightarrow 2\|T x-T y\|^{2} \leq 2\langle x-y, J(T x-T y)\rangle \\
& \Longleftrightarrow 2\|T x-T y\|^{2} \leq\|x-y\|^{2}+\|T x-T y\|^{2}-\phi(x-y, T x-T y),
\end{aligned}
$$

where

$$
\phi(x, y)=\|x\|^{2}-2\langle x, J y\rangle+\|y\|^{2}
$$

for $x, y \in E$. So, from a firmly nonexpansive mapping $T: C \rightarrow E$, we can define a nonexpansive mapping. That is, a mapping $T: C \rightarrow E$ is called nonexpansive if

$$
\|T x-T y\| \leq\|x-y\|
$$

for all $x, y \in C$. In the case that $E$ is a Hilbert space, an operator $T: C \rightarrow E$ is nonexpansive if and only if

$$
2\|T x-T y\|^{2} \leq\|x-T y\|^{2}+\|y-T x\|^{2}-2\langle x-T x, y-T y\rangle
$$

for all $x, y \in C$; see $[52,56]$.

An operator $T: C \rightarrow E$ is firmly metric if

$$
0 \leq\langle T x-T y, J(x-T x)-J(y-T y)\rangle
$$

for all $x, y \in C$. Using

$$
2\langle x-y, J z-J w\rangle=\phi(x, w)+\phi(y, z)-\phi(x, z)-\phi(y, w)
$$


for $x, y, z, w \in E$, we have that for any $x, y \in C$,

$$
\begin{aligned}
0 \leq & \langle T x-T y, J(x-T x)-J(y-T y)\rangle \\
\Longleftrightarrow & 02\langle T x-T y, J(x-T x)-J(y-T y)\rangle \\
\Longleftrightarrow & 2\langle x-T x-(y-T y), J(x-T x)-J(y-T y)\rangle \\
& \leq 2\langle x-y, J(x-T x)-J(y-T y)\rangle \\
& \quad \phi(x-T x, y-T y)+\phi(y-T y, x-T x) \\
& \leq \phi(x, y-T y)+\phi(y, x-T x)-\phi(x, x-T x)-\phi(y, y-T y) \\
\Longrightarrow & \phi(x-T x, y-T y)+\phi(y-T y, x-T x) \\
& \leq \phi(x, y-T y)+\phi(y, x-T x) .
\end{aligned}
$$

So, from a firmly metric operator, we can define a metric operator. That is, $T$ : $C \rightarrow E$ is called a metric operator if

$$
\phi(x-T x, y-T y)+\phi(y-T y, x-T x) \leq \phi(x, y-T y)+\phi(y, x-T x)
$$

for all $x, y \in C$. In the case that $E$ is a Hilbert space, $T: C \rightarrow E$ is firmly nonexpansive if

$$
\|T x-T y\|^{2} \leq\langle T x-T y, x-y\rangle
$$

for all $x, y \in C$. Further, $T: C \rightarrow E$ is a metric operator if for any $x, y \in C$,

$$
2\|x-T x-(y-T y)\|^{2} \leq\|x-(y-T y)\|^{2}+\|y-(x-T y)\|^{2} .
$$

This inequality is equivalent to

$$
\|T x-T y\|^{2} \leq 2\langle x-y, T x-T y\rangle+2\langle T x, T y\rangle .
$$

An operator $T: C \rightarrow E$ is firmly relatively nonexpansive if

$$
0 \leq\langle T x-T y, J x-J T x-(J y-J T y)\rangle
$$

for all $x, y \in C$. Then, we have that for any $x, y \in C$,

$$
\begin{aligned}
0 & \leq\langle T x-T y, J x-J T x-(J y-J T y)\rangle \\
& \Longleftrightarrow\langle T x-T y, J T x-J T y\rangle \leq\langle T x-T y, J x-J y\rangle \\
& \Longleftrightarrow \phi(T x, T y)+\phi(T y, T x) \\
& \leq \phi(T x, y)+\phi(T y, x)-\phi(T x, x)-\phi(T y, y) .
\end{aligned}
$$

So, from a firmly relatively nonexpansive operator, we can define a nonspreading operator. That is, $T: C \rightarrow E$ is a nonspreading operator [26] if

$$
\phi(T x, T y)+\phi(T y, T x) \leq \phi(T x, y)+\phi(T y, x)
$$


for all $x, y \in C$. In the case that $E$ is a Hilbert space, an operator $T: C \rightarrow E$ is firmly nonexpansive if

$$
2\|T x-T y\|^{2} \leq\|T x-y\|^{2}+\|T y-x\|^{2}-\|T x-x\|^{2}-\|T y-y\|^{2}
$$

for all $x, y \in C$. Further, an operator $T: C \rightarrow E$ is nonspreading if

$$
2\|T x-T y\|^{2} \leq\|T x-y\|^{2}+\|T y-x\|^{2}
$$

for all $x, y \in C$. This inequality is equivalent to

$$
\|T x-T y\|^{2} \leq\|x-y\|^{2}+2\langle x-T x, y-T y\rangle
$$

for all $x, y \in C$; see [14].

An operator $T: C \rightarrow E$ is firmly generalized nonexpansive if

$$
0 \leq\langle x-T x-(y-T y), J T x-J T y\rangle
$$

for all $x, y \in C$. Then, we have that for any $x, y \in C$,

$$
\begin{aligned}
0 & \leq\langle x-T x-(y-T y), J T x-J T y\rangle \\
& \Longleftrightarrow\langle T x-T y, J T x-J T y\rangle \leq\langle x-y, J T x-J T y\rangle \\
& \Longleftrightarrow \phi(T x, T y)+\phi(T y, T x) \\
& \leq \phi(x, T y)+\phi(y, T x)-\phi(x, T x)-\phi(y, T y) .
\end{aligned}
$$

So, from a firmly generalized nonexpansive operator, we can define a generalized nonexpansive type operator. That is, $T: C \rightarrow E$ is a generalized nonexpansive type operator if

$$
\phi(T x, T y)+\phi(T y, T x) \leq \phi(x, T y)+\phi(y, T x)
$$

for all $x, y \in C$.

The following is Kohsaka and Takahashi's fixed point theorem [26].

Theorem 4.1. (Kohsaka and Takahashi [26]). Let E be a smooth, strictly convex, and reflexive Banach space and let $C$ is a closed convex subset of $E$. Suppose that $T: C \rightarrow C$ is nonspreading, i.e., for all $x, y \in C$,

$$
\phi(T x, T y)+\phi(T y, T x) \leq \phi(T x, y)+\phi(T y, x) .
$$

Then the following are equivalent:

(1) There exists $x \in C$ such that $\left\{T^{n} x\right\}$ is bounded;

(2) $F(T)$ is nonempty. 
In the case that $E$ is a Hilbert space, we have the following theorem.

Theorem 4.2. (Kohsaka and Takahashi [26]). Let $H$ be a Hilbert space and let $C$ be a closed convex subset of $H$. Suppose that $T: C \rightarrow C$ is nonspreading, i.e., for all $x, y \in C$,

$$
2\|T x-T y\|^{2} \leq\|T x-y\|^{2}+\|T y-x\|^{2} .
$$

Then the following are equivalent:

(1) There exists $x \in C$ such that $\left\{T^{n} x\right\}$ is bounded;

(2) $F(T)$ is nonempty.

In the remainder of this section, we deal with nonlinear operators with fixed points in a Banach space. Let $E$ be a reflexive, smooth and strictly convex Banach space. Let $C$ be a nonempty subset of $E$. A mapping $T: C \rightarrow E$ is nonexpansive if

$$
\|T x-T y\| \leq\|x-y\|
$$

for all $x, y \in C$. A mapping $T: C \rightarrow E$ is quasi-nonexpansive if $F(T) \neq \emptyset$ and

$$
\|T x-y\| \leq\|x-y\|
$$

for all $x \in C$ and $y \in F(T)$. If $C$ is a closed convex subset of $E$ and $T: C \rightarrow C$ is quasi-nonexpansive, then $F(T)$ is closed and convex; see Itoh and Takahashi [16].

A mapping $T: C \rightarrow E$ is metric if

$$
\phi(x-T x, y-T y)+\phi(y-T y, x-T x) \leq \phi(x, y-T y)+\phi(y, x-T x)
$$

for all $x, y \in C$. A mapping $T: C \rightarrow E$ is quasi-metric if $F(T) \neq \emptyset$ and

$$
2\|x-T x\|^{2} \leq\|x\|^{2}+\phi(y, x-T x)
$$

for all $x \in C$ and $y \in F(T)$.

A mapping $T: C \rightarrow E$ is nonspreading if

$$
\phi(T x, T y)+\phi(T y, T x) \leq \phi(T x, y)+\phi(T y, x)
$$

for all $x, y \in C$. A mapping $T: C \rightarrow E$ is quasi-nonspreading or quasi-relatively nonexpansive if $F(T) \neq \emptyset$ and

$$
\phi(y, T x) \leq \phi(y, x)
$$

for all $x \in C$ and $y \in F(T)$. 
A mapping $T: C \rightarrow E$ is generalized nonexpansive type if

$$
\phi(T x, T y)+\phi(T y, T x) \leq \phi(x, T y)+\phi(y, T x)
$$

for all $x, y \in C$. A mapping $T: C \rightarrow E$ is generalized nonexpansive [12] or quasi-generalized nonexpansive type if $F(T) \neq \emptyset$ and

$$
\phi(T x, y) \leq \phi(x, y)
$$

for all $x \in C$ and $y \in F(T)$.

\section{Duality Theorems}

Let $E$ be a reflexive, smooth and strictly convex Banach space. Let $C$ be a nonempty subset of $E$. Let $T: C \rightarrow C$ be a mapping. Then, $p \in C$ is called an asymptotic fixed point of $T$ [37] if there exists $\left\{x_{n}\right\} \subset C$ such that $x_{n} \rightarrow p$ and $\lim _{n \rightarrow \infty}\left\|x_{n}-T x_{n}\right\|=0$. We denote by $\hat{F}(T)$ the set of asymptotic fixed points of $T$. Matsushita and Takahashi [28] also gave the following definition: An operator $T: C \rightarrow E$ is relatively nonexpansive if $F(T) \neq \emptyset, \hat{F}(T)=F(T)$ and

$$
\phi(y, T x) \leq \phi(y, x)
$$

for all $x \in C$ and $y \in F(T)$.

The following theorems are in Kohsaka and Takahashi [26].

Theorem 5.1. (Kohsaka and Takahashi [26]). Let E be a smooth and strictly convex Banach space and let $C$ be a closed convex subset of E. Suppose that T: $C \rightarrow C$ is nonspreading, i.e.,

$$
\phi(T x, T y)+\phi(T y, T x) \leq \phi(T x, y)+\phi(T y, x)
$$

for all $x, y \in C$. Then, $F(T)$ is closed and convex.

Theorem 5.2. (Kohsaka and Takahashi [26]). Let E be a strictly convex Banach space whose norm is uniformly Gateaux differentiable and let $C$ be a closed convex subset of E. Suppose $T: C \rightarrow C$ is nonspreading, i.e.,

$$
\phi(T x, T y)+\phi(T y, T x) \leq \phi(T x, y)+\phi(T y, x)
$$

for all $x, y \in C$. Then, $\hat{F}(T)=F(T)$. Further, if $F(T)$ is nonempty, then, $T: C \rightarrow C$ is relatively nonexpansive.

Let $E$ be a smooth Banach space and let $C$ be a nonempty subset of $E$. Let $T$ : $C \rightarrow C$ be a mapping. Then, $p \in C$ is called a generalized asymptotic fixed point of 
$T$ [13] if there exists $\left\{x_{n}\right\} \subset C$ such that $J x_{n} \rightarrow J p, \lim _{n \rightarrow \infty}\left\|J x_{n}-J T x_{n}\right\|=0$. We denote by $\check{F}(T)$ the set of generalized asymptotic fixed points of $T$.

Let $E$ be a smooth, strictly convex, and reflexive Banach space and let $C$ be a nonempty subset of $E$. Let $T$ be a mapping of $C$ into itself. Define a mapping $T^{*}$ as follows:

$$
T^{*} x^{*}=J T J^{-1} x^{*}, \quad \forall x^{*} \in J C,
$$

where $J$ is the duality mapping on $E$ and $J^{-1}$ is the duality mapping on $E^{*}$. A mapping $T^{*}$ is called the duality mapping of $T$; see also [9]. It is easy to show that $T^{*}$ is a mapping of $J C$ into itself. In fact, for $x^{*} \in J C$, we have $J^{-1} x^{*} \in C$ and hence $T J^{-1} x^{*} \in C$. So, we have

$$
T^{*} x^{*}=J T J^{-1} x^{*} \in J C .
$$

Then, $T^{*}$ is a mapping of $J C$ into itself. Further, we define the duality mapping $T^{* *}$ of $T^{*}$ as follows:

$$
T^{* *} x=J^{-1} T^{*} J x, \quad \forall x \in C .
$$

It is easy to show that $T^{* *}$ is a mapping of $C$ into itself. In fact, for $x \in C$, we have

$$
T^{* *} x=J^{-1} T^{*} J x=J^{-1} J T J^{-1} J x=T x \in C .
$$

So, $T^{* *}$ is a mapping of $C$ into itself.

Now, we obtain the following duality theorems in a Banach space.

Theorem 5.3. Let $E$ be a smooth, strictly convex and reflexive Banach space and let $C$ be a nonempty subset of $E$. Let $T$ be a mapping of $C$ into itself and let $T^{*}$ be the duality mapping of JC into itself. Then the following hold:

(1) $J F(T)=F\left(T^{*}\right)$;

(2) $J \hat{F}(T)=\check{F}\left(T^{*}\right)$;

(3) $J \check{F}(T)=\hat{F}\left(T^{*}\right)$.

Proof. (1) We have from the definition of $T^{*}$ that

$$
\begin{aligned}
x^{*} \in J F(T) & \Longleftrightarrow J^{-1} x^{*} \in F(T) \\
& \Longleftrightarrow T J^{-1} x^{*}=J^{-1} x^{*} \\
& \Longleftrightarrow J T J^{-1} x^{*}=J J^{-1} x^{*} \\
& \Longleftrightarrow T^{*} x^{*}=x^{*} \\
& \Longleftrightarrow x^{*} \in F\left(T^{*}\right) .
\end{aligned}
$$

This implies that $J F(T)=F\left(T^{*}\right)$. 
(2) Let $x^{*} \in J \hat{F}(T)$. Then $J^{-1} x^{*} \in \hat{F}(T)$. Since $J^{-1} x^{*}$ is an asymptotic fixed point of $T$, there exists a sequence $\left\{x_{n}\right\} \subset C$ such that $x_{n} \rightarrow J^{-1} x^{*}$ and $\lim _{n \rightarrow \infty}\left\|x_{n}-T x_{n}\right\|=0$. Put $x_{n}^{*}=J x_{n}$ for each $n \in \mathbb{N}$. Then, we have that $J^{-1} x_{n}^{*}=x_{n} \rightarrow J^{-1} x^{*}$ and

$$
\begin{aligned}
\left\|J^{-1} x_{n}^{*}-J^{-1} T^{*} x_{n}^{*}\right\| & =\left\|J^{-1} J x_{n}-J^{-1} J T J^{-1} J x_{n}\right\| \\
& =\left\|x_{n}-T x_{n}\right\| \rightarrow 0
\end{aligned}
$$

Since the duality mapping $J_{*}$ on $E^{*}$ is $J^{-1}$, we have $x^{*} \in \check{F}\left(T^{*}\right)$. This implies that $J \hat{F}(T) \subset \check{F}\left(T^{*}\right)$. Conversely, let $x^{*} \in \check{F}\left(T^{*}\right)$. Then, there exists a sequence $\left\{x_{n}^{*}\right\} \subset J C$ such that $J^{-1} x_{n}^{*} \rightarrow J^{-1} x^{*}$ and $\lim _{n \rightarrow \infty}\left\|J^{-1} x_{n}^{*}-J^{-1} T^{*} x_{n}^{*}\right\|=0$. Put $x_{n}=J^{-1} x_{n}^{*}$ for each $n \in \mathbb{N}$. Then, we have that $x_{n} \rightarrow J^{-1} x^{*}$ and

$$
\begin{aligned}
\left\|x_{n}-T x_{n}\right\| & =\left\|J^{-1} x_{n}^{*}-J^{-1} J T J^{-1} x_{n}^{*}\right\| \\
& =\left\|J^{-1} x_{n}^{*}-J^{-1} T^{*} x_{n}^{*}\right\| \rightarrow 0 .
\end{aligned}
$$

Hence, we have $J^{-1} x^{*} \in \hat{F}(T)$. So, we have $x^{*} \in J \hat{F}(T)$. This implies that $\check{F}\left(T^{*}\right) \subset J \hat{F}(T)$. Then, $J \hat{F}(T)=\check{F}\left(T^{*}\right)$.

(3) Let $x^{*} \in J \check{F}(T)$. Then, $J^{-1} x^{*} \in \check{F}(T)$. So, there exists a sequence $\left\{x_{n}\right\} \subset C$ such that $J x_{n} \rightarrow J J^{-1} x^{*}=x^{*}$ and $\lim _{n \rightarrow \infty}\left\|J x_{n}-J T x_{n}\right\|=0$. Put $x_{n}^{*}=J x_{n}$ for each $n \in \mathbb{N}$. Then, we have that $x_{n}^{*} \rightarrow x^{*}$ and

$$
\begin{aligned}
\left\|x_{n}^{*}-T^{*} x_{n}^{*}\right\| & =\left\|J x_{n}-J T J^{-1} J x_{n}\right\| \\
& =\left\|J x_{n}-J T x_{n}\right\| \rightarrow 0 .
\end{aligned}
$$

Hence, we have $x^{*} \in \hat{F}\left(T^{*}\right)$. This implies that $J \check{F}(T) \subset \hat{F}\left(T^{*}\right)$. Conversely, let $x^{*} \in \hat{F}\left(T^{*}\right)$. Then, there exists a sequence $\left\{x_{n}^{*}\right\} \subset J C$ such that $x_{n}^{*} \rightarrow x^{*}$ and $\lim _{n \rightarrow \infty}\left\|x_{n}^{*}-T^{*} x_{n}^{*}\right\|=0$. Put $x_{n}=J^{-1} x_{n}^{*}$ for each $n \in \mathbb{N}$. Then, we have that $J x_{n} \rightarrow x^{*}=J J^{-1} x^{*}$ and

$$
\begin{aligned}
\left\|J x_{n}-J T x_{n}\right\| & =\left\|J J^{-1} x_{n}^{*}-J T J^{-1} x_{n}^{*}\right\| \\
& =\left\|x_{n}^{*}-T^{*} x_{n}^{*}\right\| \rightarrow 0 .
\end{aligned}
$$

Hence, we have $J^{-1} x^{*} \in \check{F}(T)$. So, we have $x^{*} \in J \check{F}(T)$. This implies that $\hat{F}\left(T^{*}\right) \subset J \check{F}(T)$. Then, $J \check{F}(T)=\hat{F}\left(T^{*}\right)$.

Theorem 5.4. Let $E$ be a smooth, strictly convex and reflexive Banach space and let $C$ be a nonempty subset of $E$. Let $T$ be a nonspreading mapping of $C$ into itself and let $T^{*}$ be the duality mapping of $T$. Then $T^{*}$ is a generalized nonexpansive type mapping of JC into itself. 
Proof. Since $T^{*}=J T J^{-1}$, we have that for $x, y \in C, x^{*}=J x$ and $y^{*}=J y$,

$$
\begin{aligned}
& \phi_{*}\left(T^{*} x^{*}, T^{*} y^{*}\right)+\phi_{*}\left(T^{*} y^{*}, T^{*} x^{*}\right) \\
= & \phi_{*}\left(J T J^{-1} J x, J T J^{-1} J y\right)+\phi_{*}\left(J T J^{-1} J y, J T J^{-1} J x\right) \\
= & \phi_{*}(J T x, J T y)+\phi_{*}(J T y, J T x) \\
= & \|J T x\|^{2}-2\left\langle J T x, J^{-1} J T y\right\rangle+\|J T y\|^{2} \\
& +\|J T y\|^{2}-2\left\langle J T y, J^{-1} J T x\right\rangle+\|J T x\|^{2} \\
= & \|T x\|^{2}-2\langle J T x, T y\rangle+\|T y\|^{2}+\|T y\|^{2}-2\langle J T y, T x\rangle+\|T x\|^{2} \\
= & \phi(T y, T x)+\phi(T x, T y)
\end{aligned}
$$

and

$$
\begin{aligned}
& \phi_{*}\left(y^{*}, T^{*} x^{*}\right)+\phi_{*}\left(x^{*}, T^{*} y^{*}\right) \\
= & \phi_{*}\left(J y, J T J^{-1} J x\right)+\phi_{*}\left(J x, J T J^{-1} J y\right) \\
= & \phi_{*}(J y, J T x)+\phi_{*}(J x, J T y) \\
= & \|J y\|^{2}-2\left\langle J y, J^{-1} J T x\right\rangle+\|J T x\|^{2} \\
& +\|J x\|^{2}-2\left\langle J x, J^{-1} J T y\right\rangle+\|J T y\|^{2} \\
= & \|y\|^{2}-2\langle J y, T x\rangle+\|T x\|^{2}+\|x\|^{2}-2\langle J x, T y\rangle+\|T y\|^{2} \\
= & \phi(T x, y)+\phi(T y, x) .
\end{aligned}
$$

Since $T$ be a nonspreading mapping, we have

$$
\begin{aligned}
& \phi_{*}\left(T^{*} x^{*}, T^{*} y^{*}\right)+\phi_{*}\left(T^{*} y^{*}, T^{*} x^{*}\right) \\
= & \phi(T y, T x)+\phi(T x, T y) \\
\leq & \phi(T x, y)+\phi(T y, x) \\
= & \phi_{*}\left(y^{*}, T^{*} x^{*}\right)+\phi_{*}\left(x^{*}, T^{*} y^{*}\right) .
\end{aligned}
$$

So, $T^{*}$ is a generalized nonexpansive type mapping.

Theorem 5.5. Let $E$ be a smooth, strictly convex and reflexive Banach space and let $C$ be a nonempty subset of $E$. Let $T$ be a generalized nonexpansive type mapping of $C$ into itself and let $T^{*}$ be the duality mapping of $T$. Then $T^{*}$ is a nonspreading mapping of JC into itself.

Proof. As in the proof of Theorem 5.4, we obtain Theorem 5.5.

Using such duality theorems, we prove the following theorem which will be used in Section 6. 
Theorem 5.6. Let $E$ be a smooth and reflexive Banach space and $E^{*}$ has a uniformly Gateaux differentiable norm. Let $C$ be a closed subset of $E$ such that $J C$ is closed and convex and let $T: C \rightarrow C$ be a gneralized nonexpansive type mapping, i.e.,

$$
\phi(T x, T y)+\phi(T y, T x) \leq \phi(x, T y)+\phi(y, T x)
$$

for all $x, y \in C$. Then, the following hold:

(1) $\check{F}(T)=F(T)$;

(2) $J F(T)$ is closed and convex;

(3) $F(T)$ is closed.

Proof. Since $T$ is a mapping of $C$ into itself, we can define the duality mapping $T^{*}$ of $J C$ into itself. From Theorem 5.5, we have that $T^{*}$ is a nonspreading mapping of $J C$ into itself.

(1) From Theorem 5.2, we know $\hat{F}\left(T^{*}\right)=F\left(T^{*}\right)$. Since $J F(T)=F\left(T^{*}\right)$ and $J \check{F}(T)=\hat{F}\left(T^{*}\right)$ from Theorem 5.3, we have that $J F(T)=J \breve{F}(T)$. Since the duality mapping $J$ is one-to-one and onto, we have $\check{F}(T)=F(T)$.

(2) Since $J F(T)=F\left(T^{*}\right)$ from Theorem 5.3 and $F\left(T^{*}\right)$ is closed and convex from Theorem 5.1, we have that $J F(T)$ is closed and convex.

(3) Since $E$ is a smooth Banach space, the duality mapping $J$ is norm-to-weak* continuous. Since a closed convex set is weakly closed and $J F(T)$ is closed and convex from (2), $J F(T)$ is wealky closed. So, we obtain that $F(T)=J^{-1} J F(T)$ is closed.

\section{Strong Convergence Theorems}

In this section, using the hybrid method by Nakajo and Takahashi [33], we first prove a strong convergence theorem for generalized nonexpansive type mappings with equilibrium problems in a Banach space.

Theorem 6.1. Let $E$ be a uniformly convex and uniformly smooth Banach space and let $C$ be a closed subset of $E$ such that $J C$ is closed and convex. Let $f: J C \times J C \rightarrow \mathbb{R}$ be a bifunction satisfying (A1), (A2), (A3) and (A4) and let $S$ be a generalized nonexpansive type mapping of $C$ into itself such that $E P(f) \cap F(S) \neq \emptyset$. Let $\left\{x_{n}\right\} \subset C$ be a sequence generated by $x_{0}=x \in C$ and

$$
\left\{\begin{array}{l}
f\left(J z_{n}, J y\right)+\frac{1}{\lambda_{n}}\left\langle z_{n}-x_{n}, J y-J z_{n}\right\rangle \geq 0, \quad \forall y \in C, \\
y_{n}=\alpha_{n} x_{n}+\left(1-\alpha_{n}\right) S z_{n}, \\
C_{n}=\left\{z \in C: \phi\left(y_{n}, z\right) \leq \phi\left(x_{n}, z\right)\right\} \\
Q_{n}=\left\{z \in C:\left\langle J x_{n}-J z, x-x_{n}\right\rangle \geq 0\right\}, \\
x_{n+1}=R_{C_{n} \cap Q_{n}} x, \quad \forall n \in\{0\} \cup \mathbb{N},
\end{array}\right.
$$


where $R_{C_{n} \cap Q_{n}}$ is the sunny generalized nonexpansive retraction of $E$ onto $C_{n} \cap Q_{n}$, and $\left\{\alpha_{n}\right\} \subset[0,1]$ and $\left\{\lambda_{n}\right\} \subset[0, \infty)$ satisfy

$$
0 \leq \alpha_{n} \leq a<1 \text { and } 0<b \leq \lambda_{n}
$$

for some $a, b \in \mathbb{R}$. Then, $\left\{x_{n}\right\}$ converges strongly to $z_{0}=R_{F(S) \cap E P(f)} x$, where $R_{F(S) \cap E P(f)}$ is the sunny generalized nonexpansive retraction of $E$ onto $F(S) \cap$ $E P(f)$.

Proof. We first show that $F(S) \cap E P(f)$ is a sunny generalized nonexpansive retract of $E$. Using Lemma 2.5 and Theorem 5.6, we have that $J F(S)$ and $J E P(f)$ are closed and convex. Sinece $E$ is uniformly convex, $J$ is injective and hence

$$
J(F(S) \cap E P(f))=J F(S) \cap J E P(f),
$$

which is also closed and convex. Using Theorem 3.2, we have that $F(S) \cap E P(f)$ is a sunny generalized nonexpansive retract of $E$. Since $E$ is reflexive, $J$ is surjective and hence

$$
J Q_{n}=\left\{z^{*} \in J C:\left\langle x-x_{n}, z^{*}-J x_{n}\right\rangle \leq 0\right\}
$$

and

$$
J C_{n}=\left\{z^{*} \in J C: \phi_{*}\left(z^{*}, J y_{n}\right) \leq \phi_{*}\left(z^{*}, J x_{n}\right)\right\}
$$

for all $n \in\{0\} \cup \mathbb{N}$. We can see that $J C_{n}$ is convex since

$$
\begin{aligned}
& \phi\left(y_{n}, z\right) \leq \phi\left(x_{n}, z\right) \\
\Longleftrightarrow & \left\|y_{n}\right\|^{2}-\left\|x_{n}\right\|^{2}-2\left\langle y_{n}-x_{n}, J z\right\rangle \leq 0 .
\end{aligned}
$$

Since $J$ is injective,

$$
J\left(C_{n} \cap Q_{n}\right)=J C_{n} \cap J Q_{n} .
$$

Thus, $J C_{n}, J Q_{n}$ and $J\left(C_{n} \cap Q_{n}\right)$ are closed and convex for all $n \in\{0\} \cup \mathbb{N}$. Using Theorem 3.2, we have that each $C_{n} \cap Q_{n}$ is a sunny generalized nonexpansive retract of $E$. We next show that $C_{n} \cap Q_{n}$ is nonempty. Let $z \in F(S) \cap E P(f)$. Put $z_{n}=T_{\lambda_{n}} x_{n}$ for each $n \in\{0\} \cup \mathbb{N}$. From $z=T_{\lambda_{n}} z$ and Lemma 2.5, we have that for any $n \in\{0\} \cup \mathbb{N}$,

$$
\begin{aligned}
\phi\left(z_{n}, z\right) & =\phi\left(T_{\lambda_{n}} x_{n}, z\right) \\
& \leq \phi\left(x_{n}, z\right) .
\end{aligned}
$$

Since $S$ is of generalized nonexpansive type, we have

$$
\begin{aligned}
\phi\left(y_{n}, z\right) & =\phi\left(\alpha_{n} x_{n}+\left(1-\alpha_{n}\right) S z_{n}, z\right) \\
& \leq \alpha_{n} \phi\left(x_{n}, z\right)+\left(1-\alpha_{n}\right) \phi\left(z_{n}, z\right) \\
& \leq \alpha_{n} \phi\left(x_{n}, z\right)+\left(1-\alpha_{n}\right) \phi\left(x_{n}, z\right) \\
& =\phi\left(x_{n}, z\right) .
\end{aligned}
$$


So, we have $z \in C_{n}$ and hence $F(S) \cap E P(f) \subset C_{n}$ for all $n \in\{0\} \cup \mathbb{N}$. Next, we show by induction that $F(S) \cap E P(f) \subset C_{n} \cap Q_{n}$ for all $n \in\{0\} \cup \mathbb{N}$. It is obvious that $F(S) \cap E P(f) \subset C_{0} \cap Q_{0}$. Suppose that $F(S) \cap E P(f) \subset C_{k} \cap Q_{k}$ for some $k$. From $x_{k+1}=R_{C_{k} \cap Q_{k}} x$, we have

$$
\left\langle J x_{k+1}-J z, x-x_{k+1}\right\rangle \geq 0, \quad \forall z \in C_{k} \cap Q_{k} .
$$

Since $F(S) \cap E P(f) \subset C_{k} \cap Q_{k}$, we also have

$$
\left\langle J x_{k+1}-J z, x-x_{k+1}\right\rangle \geq 0, \quad \forall z \in F(S) \cap E P(f) .
$$

This implies $F(S) \cap E P(f) \subset Q_{k+1}$. So, we have $F(S) \cap E P(f) \subset C_{k+1} \cap Q_{k+1}$. By induction, we have $F(S) \cap E P(f) \subset C_{n} \cap Q_{n}$ for all $n \in\{0\} \cup \mathbb{N}$. This means that $\left\{x_{n}\right\}$ and $\left\{z_{n}\right\}$ are well-defined.

Since $x_{n}=R_{Q_{n}} x$ by Theorem 3.5 (1) and $x_{n+1}=R_{C_{n} \cap Q_{n}} x \subset Q_{n}$, we have from Theorem 3.5 and (2.2) that

$$
\begin{aligned}
0 & \leq 2\left\langle x-x_{n}, J x_{n}-J x_{n+1}\right\rangle \\
& =\phi\left(x, x_{n+1}\right)-\phi\left(x, x_{n}\right)-\phi\left(x_{n}, x_{n+1}\right) \\
& \leq-\phi\left(x, x_{n}\right)+\phi\left(x, x_{n+1}\right) .
\end{aligned}
$$

So, we get that

$$
\phi\left(x, x_{n}\right) \leq \phi\left(x, x_{n+1}\right) .
$$

Further, since $x_{n}=R_{Q_{n}} x$ and $z \in F(S) \cap E P(f) \subset C_{n}$, from Theorem 3.3 we have

$$
\phi\left(x, x_{n}\right) \leq \phi(x, z) .
$$

So, we have that $\lim _{n \rightarrow \infty} \phi\left(x, x_{n}\right)$ exists. This implies that $\left\{x_{n}\right\}$ is bounded. Hence, $\left\{y_{n}\right\},\left\{z_{n}\right\}$ and $\left\{S z_{n}\right\}$ are also bounded. From Theorem 3.5, we have

$$
\begin{aligned}
\phi\left(x_{n}, x_{n+1}\right) & =\phi\left(R_{Q_{n}} x, x_{n+1}\right) \\
& \leq \phi\left(x, x_{n+1}\right)-\phi\left(x, R_{Q_{n}} x\right) \\
& =\phi\left(x, x_{n+1}\right)-\phi\left(x, x_{n}\right) \rightarrow 0 .
\end{aligned}
$$

So, we have that

$$
\phi\left(x_{n}, x_{n+1}\right) \rightarrow 0 .
$$

From $x_{n+1} \in C_{n}$, we have that $\phi\left(y_{n}, x_{n+1}\right) \leq \phi\left(x_{n}, x_{n+1}\right)$. So, we get that $\phi\left(y_{n}, x_{n+1}\right) \rightarrow 0$. From Theorem 2.3, we have

$$
\lim _{n \rightarrow \infty}\left\|y_{n}-x_{n+1}\right\|=\lim _{n \rightarrow \infty}\left\|x_{n}-x_{n+1}\right\|=0 .
$$


So, we have

$$
\left\|y_{n}-x_{n}\right\| \leq\left\|y_{n}-x_{n+1}\right\|+\left\|x_{n+1}-x_{n}\right\| \rightarrow 0 .
$$

From $\left\|x_{n}-y_{n}\right\|=\left\|x_{n}-\alpha_{n} x_{n}-\left(1-\alpha_{n}\right) S z_{n}\right\|=\left(1-\alpha_{n}\right)\left\|x_{n}-S z_{n}\right\|$, we also have that

$$
\left\|S z_{n}-x_{n}\right\| \rightarrow 0
$$

Let $z \in F(S) \cap E P(f)$. Using $z_{n}=T_{\lambda_{n}} x_{n}$ and Lemma 2.5, we have that

$$
\begin{aligned}
\phi\left(x_{n}, z\right) & \geq \phi\left(x_{n}, T_{\lambda_{n}} x_{n}\right)+\phi\left(T_{\lambda_{n}} x_{n}, z\right) \\
& =\phi\left(x_{n}, z_{n}\right)+\phi\left(z_{n}, z\right)
\end{aligned}
$$

and hence

$$
\phi\left(x_{n}, z_{n}\right) \leq \phi\left(x_{n}, z\right)-\phi\left(z_{n}, z\right) .
$$

From the definition of $\phi$, we have $\phi\left(y_{n}, z\right) \leq \alpha_{n} \phi\left(x_{n}, z\right)+\left(1-\alpha_{n}\right) \phi\left(z_{n}, z\right)$ and hence

$$
\phi\left(z_{n}, z\right) \geq \frac{\phi\left(y_{n}, z\right)-\alpha_{n} \phi\left(x_{n}, z\right)}{1-\alpha_{n}} .
$$

Therefore, we have

$$
\begin{aligned}
\phi\left(x_{n}, z_{n}\right) & \leq \phi\left(x_{n}, z\right)-\frac{\phi\left(y_{n}, z\right)-\alpha_{n} \phi\left(x_{n}, z\right)}{1-\alpha_{n}} \\
& =\frac{\phi\left(x_{n}, z\right)-\phi\left(y_{n}, z\right)}{1-\alpha_{n}} .
\end{aligned}
$$

We also have

$$
\begin{aligned}
\phi\left(x_{n}, z\right)-\phi\left(y_{n}, z\right) & =\left\|x_{n}\right\|^{2}-2\left\langle x_{n}, J z\right\rangle+\|z\|^{2}-\left\|y_{n}\right\|^{2}+2\left\langle y_{n}, J z\right\rangle-\|z\|^{2} \\
& =\left\|x_{n}\right\|^{2}-\left\|y_{n}\right\|^{2}-2\left\langle x_{n}-y_{n}, J z\right\rangle \\
& \leq\left|\left\|x_{n}\right\|^{2}-\left\|y_{n}\right\|^{2}\right|+2\left|\left\langle x_{n}-y_{n}, J z\right\rangle\right| \\
& \leq\left\|x_{n}-y_{n}\right\|\left(\left\|x_{n}\right\|+\left\|y_{n}\right\|\right)+2\left\|x_{n}-y_{n}\right\|\|J z\| .
\end{aligned}
$$

Since $\phi\left(x_{n}, z\right)-\phi\left(y_{n}, z\right) \geq 0$ and $\lim _{n \rightarrow \infty}\left\|x_{n}-y_{n}\right\|=0$, we have

$$
\lim _{n \rightarrow \infty}\left(\phi\left(x_{n}, z\right)-\phi\left(y_{n}, z\right)\right)=0 .
$$

Since $0 \leq \alpha_{n} \leq a<1$, from (6.7) we have $\lim _{n \rightarrow \infty} \phi\left(x_{n}, z_{n}\right)=0$. From Theorem 2.3, we have

$$
\left\|x_{n}-z_{n}\right\| \rightarrow 0 .
$$


Since $y_{n}=\alpha_{n} x_{n}+\left(1-\alpha_{n}\right) S z_{n}$, we have $y_{n}-S z_{n}=\alpha_{n}\left(x_{n}-S z_{n}\right)$. So, from (6.6) we have

$$
\left\|y_{n}-S z_{n}\right\|=\alpha_{n}\left\|x_{n}-S z_{n}\right\| \rightarrow 0
$$

Since

$$
\left\|z_{n}-S z_{n}\right\| \leq\left\|z_{n}-x_{n}\right\|+\left\|x_{n}-y_{n}\right\|+\left\|y_{n}-S z_{n}\right\|,
$$

from (6.5), (6.8) and (6.9) we have

$$
\left\|z_{n}-S z_{n}\right\| \rightarrow 0
$$

Since $E$ is uniformly smooth, $J$ is norm-to-norm continuous. So, we have

$$
\left\|J z_{n}-J S z_{n}\right\| \rightarrow 0 .
$$

Since $\left\{J x_{n}\right\}$ is bounded, there exists a subsequence $\left\{x_{n_{i}}\right\} \subset\left\{x_{n}\right\}$ such that $J x_{n_{i}} \rightarrow z^{*}$. Since $J$ is uniformly norm-to-norm continuous on bounded sets, we have from (6.8) that

$$
\lim _{n \rightarrow \infty}\left\|J x_{n}-J z_{n}\right\|=0 .
$$

From $J x_{n_{i}} \rightarrow z^{*}$, we have $J z_{n_{i}} \rightarrow z^{*}$. From (6.11), we have $J^{-1} z^{*} \in \check{F}(S)$. Putting $z=J^{-1} z^{*}$, from Theorem 5.6 we have $z \in F(S)$. Next, let us show $z \in E P(f)$. Since $z_{n}=T_{\lambda_{n}} x_{n}$, we have, for any $y \in C$,

$$
f\left(J z_{n}, J y\right)+\frac{1}{\lambda_{n}}\left\langle J y-J z_{n}, z_{n}-x_{n}\right\rangle \geq 0 .
$$

From (A2), we have

$$
\frac{1}{\lambda_{n}}\left\langle J y-J z_{n}, z_{n}-x_{n}\right\rangle \geq f\left(J y, J z_{n}\right) .
$$

From $0<b \leq \lambda_{n}$ and (6.8), we have

$$
\lim _{n \rightarrow \infty} \frac{z_{n}-x_{n}}{\lambda_{n}}=0 .
$$

So, from (A4) we have

$$
0 \geq f\left(J y, z^{*}\right) .
$$

Put $z_{t}^{*}=t J y+(1-t) z^{*}$ for all $t \in(0,1]$ and $y \in C$. Since $J C$ is convex, we have $z_{t}^{*} \in J C$. From (A1), (A4) and (6.12), we have

$$
\begin{aligned}
0=f\left(z_{t}^{*}, z_{t}^{*}\right) & \leq t f\left(z_{t}^{*}, J y\right)+(1-t) f\left(z_{t}^{*}, z^{*}\right) \\
& \leq t f\left(z_{t}^{*}, J y\right)
\end{aligned}
$$


and hence

$$
0 \leq f\left(z_{t}^{*}, J y\right) .
$$

Letting $t \rightarrow 0$, from (A3) we have that for each $y \in C$,

$$
0 \leq f\left(z^{*}, J y\right) .
$$

This implies $z \in E P(f)$. So, we have $z \in F(S) \cap E P(f)$. Put $z_{0}=R_{F(S) \cap E P(f)} x$. Since $z_{0}=R_{F(S) \cap E P(f)} x \subset C_{n} \cap Q_{n}$ and $x_{n+1}=R_{C_{n} \cap Q_{n}} x$, we have that

$$
\phi\left(x, x_{n+1}\right) \leq \phi\left(x, z_{0}\right) .
$$

Since $\|\cdot\|^{2}$ is weakly lower semicontinuous, from $J x_{n_{i}} \rightarrow J z$ we have that

$$
\begin{aligned}
\phi(x, z) & =\|x\|^{2}-2\langle x, J z\rangle+\|J z\|^{2} \\
& \leq \liminf _{i \rightarrow \infty}\left(\|x\|^{2}-2\left\langle x, J x_{n_{i}}\right\rangle+\left\|J x_{n_{i}}\right\|^{2}\right) \\
& =\liminf _{i \rightarrow \infty} \phi\left(x, x_{n_{i}}\right) \\
& \leq \phi\left(x, z_{0}\right) .
\end{aligned}
$$

From the definition of $z_{0}$, we have $z=z_{0}$. Thus, we obtain $z^{*}=J z=J z_{0}$. So, we obtain $J x_{n} \rightarrow J z_{0}$. We finally show that $x_{n} \rightarrow z_{0}$. From (2.2), we have

$$
\phi\left(z_{0}, x_{n}\right)=\phi\left(z_{0}, x\right)+\phi\left(x, x_{n}\right)+2\left\langle z_{0}-x, J x-J x_{n}\right\rangle, \quad \forall n \in\{0\} \cup \mathbb{N} .
$$

Since $x_{n}=R_{Q_{n}} x$ and $z_{0} \in F(S) \cap E P(f) \subset Q_{n}$, we have

$$
\phi\left(x, x_{n}\right) \leq \phi\left(x, z_{0}\right)
$$

and hence

$$
\begin{aligned}
\limsup _{n \rightarrow \infty} \phi\left(z_{0}, x_{n}\right) & =\limsup _{n \rightarrow \infty}\left(\phi\left(z_{0}, x\right)+\phi\left(x, x_{n}\right)+2\left\langle z_{0}-x, J x-J x_{n}\right\rangle\right) \\
& \leq \limsup _{n \rightarrow \infty}\left(\phi\left(z_{0}, x\right)+\phi\left(x, z_{0}\right)+2\left\langle z_{0}-x, J x-J x_{n}\right\rangle\right) \\
& =\limsup _{n \rightarrow \infty}\left(\phi\left(z_{0}, x\right)+\phi\left(x, z_{0}\right)+2\left\langle z_{0}-x, J x-J z_{0}\right\rangle\right) \\
& =\phi\left(z_{0}, z_{0}\right)=0 .
\end{aligned}
$$

Thus, we get $\lim _{n \rightarrow \infty} \phi\left(z_{0}, x_{n}\right)=0$. So, from Theorem 2.3 we obtain $\lim _{n \rightarrow \infty} \| z_{0}-$ $x_{n} \|=0$. Hence, $\left\{x_{n}\right\}$ converges strongly to $z_{0}$. This completes the proof.

Next, using Theorem 2.4, we prove a strong convergence theorem by the shrinking projection method [53]. 
Theorem 6.2. Let $E$ be a uniformly convex and uniformly smooth Banach space and let $C$ be a closed subset of $E$ such that $J C$ is closed and convex. Let $f: J C \times J C \rightarrow \mathbb{R}$ be a bifunction satisfying (A1), (A2), (A3) and (A4) and let $S$ be a generalized nonexpansive type mapping of $C$ into itself such that $E P(f) \cap F(S) \neq \emptyset$. Let $C_{1}=C$ and let $\left\{x_{n}\right\} \subset C$ be a sequence generated by $x_{1}=x \in C$ and

$$
\left\{\begin{array}{l}
f\left(J z_{n}, J y\right)+\frac{1}{\lambda_{n}}\left\langle z_{n}-x_{n}, J y-J z_{n}\right\rangle \geq 0, \quad \forall y \in C, \\
y_{n}=\alpha_{n} x_{n}+\left(1-\alpha_{n}\right) S z_{n}, \\
C_{n+1}=\left\{z \in C_{n}: \phi\left(y_{n}, z\right) \leq \phi\left(x_{n}, z\right)\right\} \\
x_{n+1}=R_{C_{n+1}} x, \quad \forall n \in \mathbb{N},
\end{array}\right.
$$

where $R_{C_{n+1}}$ is the sunny generalized nonexpansive retraction of $E$ onto $C_{n+1}$, and $\left\{\alpha_{n}\right\} \subset[0,1]$ and $\left\{\lambda_{n}\right\} \subset[0, \infty)$ are sequences such that

$$
0 \leq \alpha_{n} \leq a<1 \text { and } 0<b \leq \lambda_{n}
$$

for some $a, b \in \mathbb{R}$. Then, $\left\{x_{n}\right\}$ converges strongly to $z_{0}=R_{F(S) \cap E P(f)} x$, where $R_{F(S) \cap E P(f)}$ is the sunny generalized nonexpansive retraction of $E$ onto $F(S) \cap$ $E P(f)$.

Proof. Put $z_{n}=T_{\lambda_{n}} x_{n}$ for each $n \in \mathbb{N}$ and take $z \in F(S) \cap E P(f)$. From $z=T_{\lambda_{n}} z$ and Lemma 2.5 , we have that for any $n \in \mathbb{N}$,

$$
\begin{aligned}
\phi\left(z_{n}, z\right) & =\phi\left(T_{\lambda_{n}} x_{n}, z\right) \\
& \leq \phi\left(x_{n}, z\right) .
\end{aligned}
$$

We shall show that $J C_{n}$ are closed and convex, and $F(S) \cap E P(f) \subset C_{n}$ for all $n \in \mathbb{N}$. It is obvious from the assumption that $J C_{1}=J C$ is closed and convex, and $F(S) \cap E P(f) \subset C_{1}$. Suppose that $J C_{k}$ is closed and convex, and $F(S) \cap E P(f) \subset C_{k}$. From the definition of $\phi$, we know that for $z \in C_{k}$,

$$
\begin{aligned}
& \phi\left(y_{k}, z\right) \leq \phi\left(x_{k}, z\right) \\
\Longleftrightarrow & \left\|y_{k}\right\|^{2}-\left\|x_{k}\right\|^{2}-2\left\langle y_{k}-x_{k}, J z\right\rangle \leq 0 .
\end{aligned}
$$

So, $J C_{k+1}$ is closed and convex. If $z \in F(S) \cap E P(f) \subset C_{k}$, then we have from (6.16) that

$$
\begin{aligned}
\phi\left(y_{n}, z\right) & =\phi\left(\alpha_{n} x_{n}+\left(1-\alpha_{n}\right) S z_{n}, z\right) \\
& \leq \alpha_{n} \phi\left(x_{n}, z\right)+\left(1-\alpha_{n}\right) \phi\left(z_{n}, z\right) \\
& \leq \alpha_{n} \phi\left(x_{n}, z\right)+\left(1-\alpha_{n}\right) \phi\left(x_{n}, z\right) \\
& =\phi\left(x_{n}, z\right) .
\end{aligned}
$$


Hence, we have $z \in C_{k+1}$. By induction, we have that $J C_{n}$ are closed and convex, and $F(S) \cap E P(f) \subset C_{n}$ for all $n \in \mathbb{N}$. Since $J C_{n}$ is closed and convex, from Lemma 3.2 there exists a unique sunny generalized nonexpansive retraction $R_{C_{n}}$ of $E$ onto $C_{n}$. We also know from Lemma 3.1 that $R_{C_{n}}$ is denoted by $J^{-1} \Pi_{J C_{n}} J$, where $J$ is the duality mapping and $\Pi_{J C_{n}}$ is the generalized projection of $E^{*}$ onto $J C_{n}$. Thus, $\left\{x_{n}\right\}$ is well-defined.

Since $\left\{J C_{n}\right\}$ is a nonincreasing sequence of nonempty closed convex subsets of $E^{*}$ with respect to inclusion, it follows that

$$
\emptyset \neq J F(S) \cap J E P(f) \subset \mathrm{M}-\lim _{n \rightarrow \infty} J C_{n}=\bigcap_{n=1}^{\infty} J C_{n} .
$$

Put $C_{0}^{*}=\bigcap_{n=1}^{\infty} J C_{n}$. Then, by Theorem 2.4 we have that $\left\{\Pi_{J C_{n+1}} J x\right\}$ converges strongly to $x_{0}^{*}=\Pi_{C_{0}^{*}} J x$. Since $E^{*}$ has a Fréchet differentiable norm, $J^{-1}$ is continuous. So, we have

$$
x_{n+1}=J^{-1} \Pi_{J C_{n+1}} J x \rightarrow J^{-1} x_{0}^{*} .
$$

To complete the proof, it is sufficient to show that $J^{-1} x_{0}^{*}=R_{F(S) \cap E P(f)} x$.

Since $x_{n}=R_{C_{n}} x$ and $x_{n+1}=R_{C_{n+1}} x \in C_{n+1} \subset C_{n}$, we have from Theorem 3.5 and (2.2) that

$$
\begin{aligned}
0 & \leq 2\left\langle x-x_{n}, J x_{n}-J x_{n+1}\right\rangle \\
& =\phi\left(x, x_{n+1}\right)-\phi\left(x, x_{n}\right)-\phi\left(x_{n}, x_{n+1}\right) \\
& \leq-\phi\left(x, x_{n}\right)+\phi\left(x, x_{n+1}\right)
\end{aligned}
$$

So, we get that

$$
\phi\left(x, x_{n}\right) \leq \phi\left(x, x_{n+1}\right) .
$$

Further, since $x_{n}=R_{C_{n}} x$ and $z \in F(S) \cap E P(f) \subset C_{n}$, from Lemma 3.3 we have

$$
\phi\left(x, x_{n}\right) \leq \phi(x, z)
$$

So, we have that $\lim _{n \rightarrow \infty} \phi\left(x, x_{n}\right)$ exists. This implies that $\left\{x_{n}\right\}$ is bounded. Hence, $\left\{y_{n}\right\},\left\{z_{n}\right\}$ and $\left\{S z_{n}\right\}$ are also bounded. From Theorem 3.5, we have

$$
\begin{aligned}
\phi\left(x_{n}, x_{n+1}\right) & =\phi\left(R_{C_{n}} x, x_{n+1}\right) \\
& \leq \phi\left(x, x_{n+1}\right)-\phi\left(x, R_{C_{n}} x\right) \\
& =\phi\left(x, x_{n+1}\right)-\phi\left(x, x_{n}\right) \rightarrow 0 .
\end{aligned}
$$


So, we have that

$$
\phi\left(x_{n}, x_{n+1}\right) \rightarrow 0 .
$$

From $x_{n+1} \in C_{n+1}$, we also have that $\phi\left(y_{n}, x_{n+1}\right) \leq \phi\left(x_{n}, x_{n+1}\right)$. So, we get that $\phi\left(y_{n}, x_{n+1}\right) \rightarrow 0$. Using Theorem 2.3, we have

$$
\lim _{n \rightarrow \infty}\left\|y_{n}-x_{n+1}\right\|=\lim _{n \rightarrow \infty}\left\|x_{n}-x_{n+1}\right\|=0 .
$$

So, we have

$$
\left\|y_{n}-x_{n}\right\| \leq\left\|y_{n}-x_{n+1}\right\|+\left\|x_{n+1}-x_{n}\right\| \rightarrow 0 .
$$

Since $\left\|x_{n}-y_{n}\right\|=\left\|x_{n}-\alpha_{n} x_{n}-\left(1-\alpha_{n}\right) S z_{n}\right\|=\left(1-\alpha_{n}\right)\left\|x_{n}-S z_{n}\right\|$ and $0 \leq \alpha_{n} \leq a<1$, we also have that

$$
\left\|S z_{n}-x_{n}\right\| \rightarrow 0 .
$$

Let $z \in F(S) \cap E P(f)$. Using $z_{n}=T_{\lambda_{n}} x_{n}$ and Lemma 2.5, we have that

$$
\begin{aligned}
\phi\left(x_{n}, z\right) & \geq \phi\left(x_{n}, T_{\lambda_{n}} x_{n}\right)+\phi\left(T_{\lambda_{n}} x_{n}, z\right) \\
& =\phi\left(x_{n}, z_{n}\right)+\phi\left(z_{n}, z\right)
\end{aligned}
$$

and hence

$$
\phi\left(x_{n}, z_{n}\right) \leq \phi\left(x_{n}, z\right)-\phi\left(z_{n}, z\right) .
$$

From the definition of $\phi$, we have $\phi\left(y_{n}, z\right) \leq \alpha_{n} \phi\left(x_{n}, z\right)+\left(1-\alpha_{n}\right) \phi\left(z_{n}, z\right)$ and hence

$$
\phi\left(z_{n}, z\right) \geq \frac{\phi\left(y_{n}, z\right)-\alpha_{n} \phi\left(x_{n}, z\right)}{1-\alpha_{n}} .
$$

Therefore, we have

$$
\begin{aligned}
\phi\left(x_{n}, z_{n}\right) & \leq \phi\left(x_{n}, z\right)-\frac{\phi\left(y_{n}, z\right)-\alpha_{n} \phi\left(x_{n}, z\right)}{1-\alpha_{n}} \\
& =\frac{\phi\left(x_{n}, z\right)-\phi\left(y_{n}, z\right)}{1-\alpha_{n}} .
\end{aligned}
$$

We also have

$$
\begin{aligned}
\phi\left(x_{n}, z\right)-\phi\left(y_{n}, z\right) & =\left\|x_{n}\right\|^{2}-2\left\langle x_{n}, J z\right\rangle+\|z\|^{2}-\left\|y_{n}\right\|^{2}+2\left\langle y_{n}, J z\right\rangle-\|z\|^{2} \\
& =\left\|x_{n}\right\|^{2}-\left\|y_{n}\right\|^{2}-2\left\langle x_{n}-y_{n}, J z\right\rangle \\
& \leq\left|\left\|x_{n}\right\|^{2}-\left\|y_{n}\right\|^{2}\right|+2\left|\left\langle x_{n}-y_{n}, J z\right\rangle\right| \\
& \leq\left\|x_{n}-y_{n}\right\|\left(\left\|x_{n}\right\|+\left\|y_{n}\right\|\right)+2\left\|x_{n}-y_{n}\right\|\|J z\| .
\end{aligned}
$$


Since $\lim _{n \rightarrow \infty}\left\|x_{n}-y_{n}\right\|=0$ from (6.21), we have

$$
\lim _{n \rightarrow \infty}\left(\phi\left(x_{n}, z\right)-\phi\left(y_{n}, z\right)\right)=0 .
$$

Since $0 \leq \alpha_{n} \leq a<1$, we have $\lim _{n \rightarrow \infty} \phi\left(x_{n}, z_{n}\right)=0$. So, from Theorem 2.3, we have

$$
\left\|x_{n}-z_{n}\right\| \rightarrow 0 .
$$

From $y_{n}=\alpha_{n} x_{n}+\left(1-\alpha_{n}\right) S z_{n}$, we have $y_{n}-S z_{n}=\alpha_{n}\left(x_{n}-S z_{n}\right)$. So, from (6.22) we have

$$
\left\|y_{n}-S z_{n}\right\|=\alpha_{n}\left\|x_{n}-S z_{n}\right\| \rightarrow 0
$$

Since

$$
\left\|z_{n}-S z_{n}\right\| \leq\left\|z_{n}-x_{n}\right\|+\left\|x_{n}-y_{n}\right\|+\left\|y_{n}-S z_{n}\right\|,
$$

from (6.21), (6.24) and (6.25) we have

$$
\left\|z_{n}-S z_{n}\right\| \rightarrow 0 \text {. }
$$

Since $E$ is uniformly smooth, $J$ is norm-to-norm continuous. So, we have

$$
\left\|J z_{n}-J S z_{n}\right\| \rightarrow 0 .
$$

Since $J x_{n}=\Pi_{J C_{n}} x \rightarrow x_{0}^{*}=J J^{-1} x_{0}^{*}$, we have $J z_{n} \rightarrow x_{0}^{*}$. So, from (6.27) and Theorem 5.6 we have $J^{-1} x_{0}^{*} \in F(S)$. Next, let us show $J^{-1} x_{0}^{*} \in E P(f)$. From $x_{n} \rightarrow J^{-1} x_{0}^{*}$ and (6.24), we have $z_{n} \rightarrow J^{-1} x_{0}^{*}$. We have from $z_{n}=T_{\lambda_{n}} x_{n}$ that for any $y \in C$,

$$
f\left(J z_{n}, J y\right)+\frac{1}{\lambda_{n}}\left\langle J y-J z_{n}, z_{n}-x_{n}\right\rangle \geq 0 .
$$

From (A2), we have

$$
\frac{1}{\lambda_{n}}\left\langle J y-J z_{n}, z_{n}-x_{n}\right\rangle \geq f\left(J y, J z_{n}\right) .
$$

From $0<b \leq \lambda_{n}$ and (6.24), we know

$$
\lim _{n \rightarrow \infty} \frac{z_{n}-x_{n}}{\lambda_{n}}=0 .
$$

So, we have

$$
0 \geq f\left(J y, x_{0}^{*}\right) .
$$


Put $z_{t}^{*}=t J y+(1-t) x_{0}^{*}$ for all $t \in(0,1]$ and $y \in C$. Since $J C$ is convex, we have $z_{t}^{*} \in J C$. From (A1), (A4) and (6.28), we have

$$
\begin{aligned}
0=f\left(z_{t}^{*}, z_{t}^{*}\right) & \leq t f\left(z_{t}^{*}, J y\right)+(1-t) f\left(z_{t}^{*}, x_{0}^{*}\right) \\
& \leq t f\left(z_{t}^{*}, J y\right)
\end{aligned}
$$

and hence

$$
0 \leq f\left(z_{t}^{*}, J y\right)
$$

Letting $t \rightarrow 0$, we have from (A3) that for each $y \in C$,

$$
0 \leq f\left(x_{0}^{*}, J y\right) .
$$

This implies $J^{-1} x_{0}^{*} \in E P(f)$. So, we have that $J^{-1} x_{0}^{*} \in F(S) \cap E P(f)$. Put $z_{0}=R_{F(S) \cap E P(f)} x$. Since $z_{0}=R_{F(S) \cap E P(f)} x \subset C_{n+1}$ and $x_{n+1}=R_{C_{n+1}} x$, we have that

$$
\phi\left(x, x_{n+1}\right) \leq \phi\left(x, z_{0}\right)
$$

So, we have that

$$
\begin{aligned}
\phi\left(x, J^{-1} x_{0}^{*}\right) & =\lim _{n \rightarrow \infty} \phi\left(x, x_{n}\right) \\
& \leq \phi\left(x, z_{0}\right) .
\end{aligned}
$$

So, we get $z_{0}=J^{-1} x_{0}^{*}$. Hence, $\left\{x_{n}\right\}$ converges strongly to $z_{0}$. This completes the proof.

\section{ACKNOWLEDGMENTS}

The first author and the second author were partially supported by Grant-in-Aid for Scientific Research No. 19540167 from Japan Society for the Promotion of Science and by the grant NSC 98-2115-M-110-001, respectively.

\section{REFERENCES}

1. Y. I. Alber, Metric and generalized projections in Banach spaces: Properties and applications, in: Theory and Applications of Nonlinear Operators of Accretive and Monotone Type, A. G. Kartsatos ed., Marcel Dekker, New York, 1996, pp. 15-20.

2. Y. I. Alber and S. Reich, An iterative method for soling a class of nonlinear operator equations in Banach spaces, PanAmer. Math. J., 4 (1994), 39-54.

3. K. Aoyama, Y. Kimura and W. Takahashi, Maximal monotone operators and maximal monotone functions for equilibrium problems, J. Convex Anal., 15 (2008), 395-409. 
4. K. Aoyama, F. Kohsaka and W. Takahashi, Three generalizations of firmly nonexpansive mappings: Their relations and continuity properties, J. Nonlinear Convex Anal., 10 (2009), 131-147.

5. K. Aoyama and W. Takahashi, Strong convergence theorems for a family of relatively nonexpansive mappings in Banach spaces, Fixed Point Theory, 8 (2007), 143-160.

6. E. Blum and W. Oettli, From optimization and variational inequalities to equilibrium problems, Math. Student, 63 (1994), 123-145.

7. P. L. Combettes and A. Hirstoaga, Equilibrium programming in Hilbert spaces, J. Nonlinear Convex Anal., 6 (2005), 117-136.

8. J. Diestel, Geometry of Banach spaces, Selected Topics, Lecture Notes in Mathematics, 485, Springer, Berlin, 1975.

9. T. Honda, T. Ibaraki and W. Takahashi, Duality theorems and convergence theorems for nonlineaqr mappings in Banach spaces, Int. J. Math. Statis., 6 (2010), 46-64.

10. T. Honda and W. Takahashi, Nonlinear projections and generalized conditional expectations in Banach spaces, Taiwanese J. Math., to appear.

11. T. Ibaraki, Y. Kimura and W. Takahashi, Convergence theorems for generalized projections and maximal monotone operators in Banach spaces, Abst. Appl. Anal., 2003 (2003), 621-629.

12. T. Ibaraki and W. Takahashi, A new projection and convergence theorems for the projections in Banach spaces, J. Approx. Theory, 149 (2007), 1-14.

13. T. Ibaraki and W. Takahashi, Generalized nonexpansive mappings and a proximaltype algorithm in Banach spaces, Contemp. Math., to appear.

14. S. Iemoto and W. Takahashi, Approximating fixed points of nonexpansive mappings and nonspreading mappings in a Hilbert space, Nonlinear Anal., 71 (2009), 20822089.

15. H. Iiduka and W. Takahashi, Weak convergence theorem by Cesaro means for nonexpansive mappings and inverse-strongly monotone mappings, J. Nonlinear Convex Anal., 7 (2006), 105-113.

16. S. Itoh and W. Takahashi, The common fixed point theory of singlevalued mappings and multivalued mappings, Pacific J. Math., 79 (1978), 493-508.

17. S. Kamimura, F. Kohsaka and W. Takahashi, Weak and strong convergence theorems for maximal monotone operators in a Banach space, Set-Valued Anal., 12 (2004), 417-429.

18. S. Kamimura and W. Takahashi, Approximating solutions of maximal monotone operators in Hilbert spaces, J. Approx. Theory, 106 (2000), 226-240.

19. S. Kamimura and W. Takahashi, Weak and strong convergence of solutions to accretive operator inclusions and applications, Set-Valued Anal., 8 (2000), 361-374.

20. S. Kamimura and W. Takahashi, Strong convergence of a proximal-type algorithm in a Banach apace, SIAM J. Optim., 13 (2002), 938-945. 
21. Y. Kimura and W. Takahashi, On a hybrid method for a family of relatively nonexpansive mappings in a Banach apace, J. Math. Anal. Appl., 357 (2009), 356-363.

22. F. Kohsaka and W. Takahashi, Strong convergence of an iterative sequence for maximal monotone operators in a Banach space, Abstr. Appl. Anal., 2004 (2004), 239249.

23. F. Kohsaka and W. Takahashi, Weak and strong convergence theorems for minimax problems in Banach spaces, in Nonlinear Analysis and Convex Analysis (W. Takahashi and T. Tanaka, eds.), Yokohama Publishers, 2004, pp. 203-216.

24. F. Kohsaka and W. Takahashi, Generalized nonexpansive retractions and a proximaltype algorithm in Banach spaces, J. Nonlinear Convex Anal., 8 (2007), 197-209.

25. F. Kohsaka and W. Takahashi, Existence and approximation of fixed points of firmly nonexpansive-type mappings in Banach spaces, SIAM J. Optim., 19 (2008), 824-835.

26. F. Kohsaka and W. Takahashi, Fixed point theorems for a class of nonlinear mappings related to maximal monotone operators in Banach spaces, Arch. Math., 91 (2008), 166-177.

27. S. Matsushita and W. Takahashi, Weak and strong convergence theorems for relatively nonexpansive mappings in Banach spaces, Fixed Point Theory Appl., 2004 (2004), $37-47$.

28. S. Matsushita and W. Takahashi, A strong convergence theorem for relatively nonexpansive mappings in a Banach space, J. Approx. Theory, 134 (2005), 257-266.

29. S. Matsushita and W. Takahashi, Approximating fixed points of nonexpansive mappings in a Banach space by metric projections, Applied Math. Comput., 196 (2008), 422-425.

30. U. Mosco, convergence of convex sets and of solutions of variational inequalities, Adv. Math., 3 (1969), 510-585.

31. A. Moudafi, Weak convergence theorems for nonexpansive mappings and equilibrium problems, J. Nonlinear Convex Anal., 9 (2008), 37-43.

32. A. Moudafi and M. Thera, Proximal and dynamical approaches to equilibrium problems, in: Lecture Notes in Economics and Mathematical Systems, 477, Springer, 1999, pp. 187-201.

33. K. Nakajo and W. Takahashi, Strong convergence theorems for nonexpansive mappings and nonexpansive semigroups, J. Math. Anal. Appl., 279 (2003), 372-379.

34. S. Ohsawa and W. Takahashi, Strong convergence theorems for resolvento of maximal monotone operator, Arch. Math., 81 (2003), 439-445.

35. S. Reich, Constructive techniques for accretive and monotone operators, Applied Nonlinear Analysis, (V. Lakshmikan, ed.), Academic Press, New York, 1979, pp. 335-345.

36. S. Reich, Strong convergence theorems for resolvents of accretive operators in Banach spaces, J. Math. Anal. Appl., 75 (1980), 287-292. 
37. S. Reich, A weak convergence theorem for the alternative method with Bregman distance, in: Theory and Applications of Nonlinear Operators of Accretive and Monotone Type (A. G. Kartsatos ed.), Marcel Dekker, New York, 1996, pp. 313-318.

38. R. T. Rockafellar Characterization of the subdifferentials of convex functions, Pacific J. Math., 17 (1966), 497-510.

39. R. T. Rockafellar, On the maximality of sums of nonlinear monotone operators, Trans. Amer. Math. Soc., 149 (1970), 75-88.

40. R. T. Rockafellar, Monotone operators and the proximal point algorithm, SIAM J. Control Optim., 14 (1976), 877-898.

41. M. V. Solodov and B. F. Svaiter, Forcing strong convergence of proximal point iterations in a Hilbert space, Math. Program., 87 (2000), 189-202.

42. A. Tada and W. Takahashi, Strong convergence theorem for an equilibrium problem and a nonexpansive mapping, J. Optim. Theory Appl., 133 (2007), 359-370.

43. S. Takahashi and W. Takahashi, Viscosity approximation methods for equilibrium problems and fixed point problems in Hilbert spaces, J. Math. Anal. Appl., 331 (2007), 506-515.

44. S. Takahashi and W. Takahashi, Strong convergence theorems for a generalized equilibrium problem anda nonexpansive mapping in a Hilbert space, Nonlinear Anal., 69 (2008), 1025-1033.

45. W. Takahashi, Iterative methods for approximation of fixed points and their applications, J. Oper. Res. Soc. Japan, 43 (2000), 87-108.

46. W. Takahashi, Nonlinear Functional Analysis, Yokohama Publishers, Yokohama, 2000.

47. W. Takahashi, Convex Analysis and Approximation of Fixed Points (Japanese), Yokohama Publishers, Yokohama, 2000.

48. W. Takahashi, Introduction to Nonlinear and Convex Analysis (Japanese), Yokohama Publishers, Yokohama, 2005.

49. W. Takahashi, Viscosity approximation methods for resolvents of accretive operators in Banach spaces, J. Fixed Point Theory Appl., 1 (2007), 135-147.

50. W. Takahashi, Proximal point algorithms and four resolvents of nonlinear operators of monotone type in Banach spaces, Taiwanese J. Math., 12 (2008), 1883-1910.

51. W. Takahashi, Viscosity approximation methods for countable families of nonexpansive mappings in Banach spaces, Nonlinear Anal., 70 (2009), 719-734.

52. W. Takahashi, Fixed point theorems for new nonexpansive mappings in a Hilbert space, J. Nonlinear Convex Anal., 11 (2010), 78-88.

53. W. Takahashi, Y. Takeuchi and R. Kubota, Strong convergence theorems by hybrid methods for families of nonexpansive mappings in Hilbert spaces, J. Math. Anal. Appl., 341 (2008), 276-286. 
54. W. Takahashi and M. Toyoda, Weak convergence theorems for nonexpansive mappings and monotone mappings, J. Optim. Theory Appl., 118 (2003), 417-428.

55. W. Takahashi and Y. Ueda, On Reich's strong convergence theorems for resolvents of accretive operators, J. Math. Anal. Appl., 104 (1984), 546-553.

56. W. Takahashi and J. C. Yao, Fixed point theorems and ergodic theorems for nonlinear mappings in a Hilbert space, Taiwanese J. Math., to appear.

57. W. Takahashi and K. Zembayashi, A strong convergence theorem for the equilibrium problem with a bifunction defined on the dual space of a Banach space, in Fixed Point Theory and its Applications (S. Dhompongsa, K. Goebel, W. A. Kirk eds.), Yokohama Publishers, Yokohama, 2008, pp. 197-209.

58. W. Takahashi and K. Zembayashi, Strong and weak convergence theorem for equilibrium problems and relatively nonexpansive mappings in Banach spaces, Nonlinear Anal., 70 (2009), 45-57.

\author{
Wataru Takahashi \\ Department of Applied Mathematics \\ National Sun Yat-sen University \\ Kaohsiung 80424, Taiwan \\ E-mail: wataru@is.titech.ac.jp \\ Jen-Chih Yao \\ Center for General Education \\ Kaohsiung Medical University \\ Kaohsiung 80707, Taiwan \\ E-mail: yaojc@math.nsysu.edu.tw
}

